\title{
Hydrogen on the rise
}

\author{
Using hydrogen as an energy carrier has long been discussed as a route to a greener future, and although \\ headway has been less significant than many hoped, recent developments point to tangible progress.
}

In light of the recent commercial successes in the battery electric vehicle (BEV) industry, it is easy to be pessimistic about the prospects of another class of electric vehicles - fuel cell electric vehicles (FCEVs), fed with hydrogen, producing only water at the exhaust. BEVs seem to have eclipsed their FCEV cousins in the public consciousness as the 'go to' option for green personal transportation, spurred on by impressive technological advances in battery energy density and reductions in cost, in addition to the roll-out of charging infrastructure.

A world powered by hydrogen has been talked about for decades, and the feeling that it is, and always will be, a fuel of the future is borne out of the seemingly slow progress that has been made, particularly in developing infrastructure and bringing down costs. However, perhaps unsurprisingly, there was certainly a sense of optimism this June, when the World Hydrogen Energy Conference convened for the 21 st time since 1976 to discuss all aspects of hydrogen technology. FCEVs of course dominated much of the discussion.

Toyota's Mirai — billed as the world's first mass-produced hydrogen fuel cell vehicle - has demonstrated its commitment to the technology. With Honda releasing its brand of FCEV to the market this year, and BMW collaborating with Toyota on fuel cell technology for their vehicles, large automobile makers are clearly not giving up on hydrogen just yet. Advocates of FCEVs quote their extensive driving range and quick refuel time - measured in hundreds of miles and single-digit minutes, respectively - as advantages over BEVs. There are other benefits, too; in a world where the amount of renewable electricity is only going to increase, the need for enhanced flexibility is vital. In this context the ability to decouple the time when electricity is generated from when it is used is of paramount importance. Electrolysis to generate hydrogen from water thus becomes attractive as surplus electricity can be used to produce hydrogen at times of low demand. FCEV s could avoid the strain on the grid that may be envisaged if the world only drove BEVs, many of which would likely be charged in the hours immediately after a city gets home from work.

Opponents to FCEVs tend to point to the lack of hydrogen refuelling infrastructure as one example of a major barrier to market penetration. However in this department, tangible progress is being made, if only in rather select regions. The number of retail refuelling stations in California is now at least 20, with more planned by the end of the year (http://cafcp.org/stationmap). These are clustered in the metropolitan areas surrounding Los Angeles and San Francisco with additional 'corridor stations' linking the two urban hubs. Elsewhere, there are plans in Germany to build 400 refuelling stations by $2023^{1}$. Similarly, Japan plans to have 160 hydrogen refuelling stations by 2020 , and 320 by $2025^{2}$. Beyond these operations, further network expansion will depend on consumer uptake of FCEVs and demand for greater infrastructure. There is certainly a long way to go; estimates suggest the number of FCEVs on California's roads today number in the hundreds - a tiny fraction of the approximately 30 million vehicles in the state.

But of course it is not just personal automobiles that can be powered by hydrogen. Recently, the northern German state of Schleswig-Holstein announced plans to electrify its entire rail network by 2025 , making use of fuel cell technology to power the trains ${ }^{3}$. The region is a net exporter of electricity, with a relatively large supply of wind, so it has excess capacity that could be used to power electrolysers to split water, highlighting how hydrogen can be a vector for an integrated energy system. The arguments for using hydrogen to power mass transit systems are also perhaps more apparent than for personal travel. Well-defined routes with fixed terminuses mean that range anxiety is not an issue and the number of refuelling stations can be reasonably small.

Plans are also afoot that demonstrate hydrogen's potential role in stationary applications. This year plans were announced to transform the UK city of Leeds into a 'hydrogen city' in which the natural gas that runs through the city's pipes would be switched to hydrogen ${ }^{4}$. Consumers would use hydrogen to heat their homes and cook their meals, requiring substantial conversion of domestic boilers and cookers. This is of course still a long way off, and does not come cheap, with costs estimated at $£ 2$ billion. The idea is attractive from an emissions perspective, if the hydrogen can be produced in a green fashion, but it is likely that production would rely on reforming of natural gas, which would require carbon capture technology to make the process carbon neutral. Nevertheless, the success of such ambitious plans would surely play a pivotal role in decisions over further projects related to hydrogen infrastructure, and allay public fears over the safety of hydrogen power. In that regard, Japan has announced plans to spend 40 billion yen to encourage hydrogen technologies in the run up to the Tokyo 2020 Olympics 5 . As part of the scheme, pipelines could run through the athletes' village, delivering hydrogen to industrial-scale fuel cells that would provide the electricity to power buildings.

We are still far from a time when hydrogen runs through our pipes on a large scale, heating our homes and powering our cars, but the visibility of schemes such as those for Tokyo 2020 may help to raise the profile of hydrogen technologies. If that encourages more people to use FCEVs, economies of scale could lead to the cost of hydrogen falling, making its use as an energy vector more appealing in multiple domains. But it is also imperative that the hydrogen can be produced in a green way. Thus, the intense research efforts into more efficient, environmentallyfriendly technologies to generate hydrogen through electrolysis and solar-driven water splitting are well-placed. Diversity and flexibility within energy systems are key our energy future will be more mixed than at present. In that regard, hydrogen may take its place alongside other energy storage technologies in its own niche, where the benefits of hydrogen power are strong for a specific application - and location. It remains to be seen quite when hydrogen technology will come of age, but there are signs that it is starting to emerge from a difficult adolescence.

\footnotetext{
References

1. Germany: H2 MOBILITY targets 400 hydrogen fueling stations by 2023. Hydrogen Mobility Europe (5 May 2016); http://go.nature.com/2alhFRa

2. Watanabe, C. Japan eyes 40,000 fuel-cell cars, 160 hydrogen stations by 2020. Bloomberg (16 March 2016); http://go.nature.com/2ag0kJV

3. Grey, E. German state thrusts hydrogen-powered hydrail into the spotlight. Railway Technology (21 June 2016); http://go.nature.com/29Ro6XT

4. Gosden, E. UK homes could be heated by hydrogen under plan to tackle global warming. The Telegraph (9 April 2016); http://go.nature.com/2alx $5 \mathrm{We}$

5. Obe, M. Tokyo hopes to make hydrogen power the star of the 2020 Olympics. The Wall Street Journal (15 September 2015); http://go.nature.com/29Yigcb
} 